\title{
Gefitinib as first line therapy in Malaysian patients with EGFR mutation-positive non-small-cell lung cancer: A single-center retrospective study
}

\author{
MATIN MELLOR ABDULLAH ${ }^{1 *}$, AMIT BHAT $^{2 *}$, AHMAD KAMAL MOHAMED $^{1}$, \\ FOO YOKE CHING $^{1}$, NIDA AHMED ${ }^{2}$ and SANDEEP GANTOTTI ${ }^{2}$
}

\author{
${ }^{1}$ Department of Clinical Oncology and Radiotherapy, Subang Jaya Medical Centre, Subang Jaya 47500, Selangor, Malaysia; \\ ${ }^{2}$ Indegene Lifesystems Pvt. Ltd., Nagavara, Karnataka 560045, Bangalore, India
}

Received February 17, 2015; Accepted February 19, 2016

DOI: $10.3892 / \mathrm{ol} .2016 .4322$

\begin{abstract}
The present retrospective, single-center study evaluated the objective response rate (ORR) and progression-free survival (PFS) of epidermal growth factor receptor (EGFR) mutation-positive Malaysian patients with advanced lung adenocarcinoma treated with gefitinib. During May 2008 to July 2013, 33 patients with Stage IV, EGFR mutation-positive non-small-cell lung cancer (NSCLC) were identified and received gefitinib $(250 \mathrm{mg})$ as first line treatment. The primary and secondary end points were ORR, PFS and safety, respectively. A total of $18(54.5 \%)$ and $2(6.1 \%)$ patients achieved partial response (PR) and complete response (CR) to gefitinib therapy, respectively, yielding an ORR of $60.6 \%$ (95\% CI, 42.1-77.1\%). Patients with exon 20 or 21 mutations $(n=6,66.7 \%)$ tended to have better ORR compared with exon 19 ( $n=22$, 59.1\%). The median PFS was 8.9 months in Malaysian patients with EGFR mutation-positive NSCLC, treated with gefitinib. The majority of treatment-related toxicity was mild in nature. The most frequently reported adverse events included dry skin $(39.4 \%)$, skin rash $(27.2 \%)$, and dermatitis acneiform $(15.2 \%)$. In conclusion, Malaysian patients with locally advanced and metastatic EGFR mutation-positive NSCLC responded favorably to gefitinib therapy in terms of ORR, median PFS, and tolerability, the results of which were consistent with those of the IPASS study conducted in an Asian population. Considering the efficacy and safety profile of gefitinib, it is a favorable option for the first-line treatment of Malaysian patients with EGFR mutation-positive NSCLC. However, future long-term
\end{abstract}

Correspondence to: Dr Matin Mellor Abdullah, Department of Clinical Oncology and Radiotherapy, Subang Jaya Medical Centre, 1 Jalan SS 12/1A, Ss 12, Subang Jaya 47500, Selangor, Malaysia

E-mail:drmatinmellor@gmail.com

${ }^{*}$ Contributed equally

Key words: non-small-cell lung carcinoma, adenocarcinoma, EGFR mutation positive, gefitinib, Malaysia studies in a larger population of Malaysian patients are required to support whether the prolonged PFS conferred by gefitinib will translate into prolonged overall survival.

\section{Introduction}

Lung cancer is the leading cause of cancer-related mortality worldwide and non-small-cell lung cancer (NSCLC) accounts for $\sim 88 \%$ of the cases (1). In Malaysia specifically, lung cancer is the third leading cause of cancer-related mortality accounting for $\sim 19.8 \%$ of cancer-related deaths (2). In spite of several advances in the treatment modalities, the burden of lung cancer in Malaysia remains high, with an incidence rate of $13.8 \%$ in males and $3.8 \%$ in females (2). Adenocarcinoma, a histological variant of NSCLC, is the most common type of cancer observed in Malaysian patients irrespective of their smoking status (1). Guidelines for the management of NSCLC strongly recommend testing for epidermal growth factor receptor (EGFR) mutation (3). This specific mutation has a predilection for Asians, females, non-smokers and patients with adenocarcinoma $(4,5)$. The presence of $E G F R$ mutation was found to be a strong predictive biomarker for the clinical efficacy of $E G F R$ tyrosine kinase inhibitors (TKI) such as gefitinib (6).

A number of studies have reported improved outcomes with gefitinib monotherapy in terms of prolonged progression-free survival (PFS) and improvements in time to treatment failure (TTF) when used as a first-line treatment in East Asian patients with advanced NSCLC positive for EGFR mutations (7-10). Gefitinib (IRESSA ${ }^{\circledR}$, AstraZeneca) is a once-daily oral medication (usually given at a dose of $250 \mathrm{mg}$ ) indicated in advanced NSCLC, for patients with EGFR mutations. IPASS (IRESSA ${ }^{\circledR}$ Pan-Asia Study) was a randomized, large-scale, double-blinded study; the study compared gefitinib versus carboplatin/paclitaxel as a first line treatment in 1,217 patients in Asia with advanced NSCLC. The IPASS study established gefitinib as a potential first-line therapy for patients with EGFR mutation-positive tumors and showed superior PFS for gefitinib over intravenous carboplatin/paclitaxel chemotherapy in clinically selected Asians with advanced NSCLC [Hazard ratio (HR) $0.74,95 \%$ confidence interval (CI) 0.65 to $0.85, \mathrm{P}<0.0001]$. IPASS also reported there was a significantly 
higher response rate with an improved tolerability profile and superior quality of life rates with gefitinib compared with carboplatin/paclitaxel chemotherapy (6).

Although data exists on the use of gefitinib in Asian population, there is very limited data on the use of this drug in patients of Malaysian descent $(11,12)$. Therefore, the present study was conducted to investigate the Malaysian experience with gefitinib from a single-center in EGFR mutation-positive NSCLC patients.

\section{Patients and methods}

Study design. The present retrospective, single-center study was conducted to evaluate the response and survival rate of Malaysian patients who had been treated with gefitinib (IRESSA ${ }^{\circledR}$, Astrazeneca, London, UK) for EGFR-positive NSCLC. The primary end point was the objective response rate (ORR). Secondary end points were PFS, and safety. This study followed the ethical principles approved by the institutional review board of the hospital. All patients' data was kept anonymous. The list of patients who had received gefitinib prior to December 2013 for their lung cancer was traced, these patients' charts were obtained for review and the appropriate data was extracted.

Patient population. All patients with Stage IV, EGFR mutation-positive NSCLC who received gefitinib as first- line treatment between May 2008 and July 2013 (Subang Jaya Medical Center, Subang Jaya, Malaysia) were identified and included in this analysis, after approval from the local ethics committee. Medical charts of the patients were reviewed. Patients were selected based on the following inclusion criteria: Male or female aged $\geq 18$ years; patients diagnosed with NSCLC, which was confirmed histologically or cytologically as adenocarcinoma or adenosquamous carcinoma; patients with Stage IV disease that was not curable with surgery or radiotherapy; and patients with an Eastern Cooperative Oncology Group (ECOG) performance status of 0-3. Patients who had a prior history of chemotherapy with other drugs such as erlotinib, carboplatin/gemcitabine, zoledronic acid and carboplatin/pemetrexed, were also included as long as they switched to gefitinib. Only patients who were EGFR negative (and hence, not given gefitinib) were excluded from this analysis. All the patients in the study underwent comprehensive baseline assessments that included clinical laboratory tests and imaging studies. Follow-up assessments and monitoring of all the patients were also carried out at weekly intervals. Toxicity evaluations followed the National Cancer Institute Common Terminology Criteria for Adverse Events, version 3.0 (v3.0). With the exception of 3 patients, all were non-smokers.

Efficacy and safety assessments. The response rate [complete response $(\mathrm{CR})$, partial response (PR), stable disease (SD), and progressive disease (PD) were recorded] were evaluated as the main outcome for the patients treated with gefitinib in the present study. Disease progression was monitored by x-rays, computerized tomography (CT), positron emission tomography (PET), magnetic resonance imaging (MRI) and bone scans. The duration of response from gefitinib was recorded in months. The adverse event (AE) profile of gefitinib was also evaluated and graded as mild (Grade 1), moderate (Grade 2), and severe (Grade 3). Patients were considered non-evaluable if they did not have adequate reports available for response rate or progression of disease. PFS was measured from Day 1 of the treatment until clinical signs of disease progression. EGFR mutation testing was performed by quantitative polymerase chain reaction (qPCR) amplification and bidirectional sequencing. All patients were confirmed as having EGFR-positive Grade IV adenocarcinoma. Diagnosis was performed in the study mainly by CT-guided lung biopsy. Other related procedures that were used to aid diagnosis were percutaneous lung biopsy, bilateral neck nodes biopsy, pleural biopsy, paraspinal mass biopsy, pleural cytology and bronchoscopic biopsy. The actual clinical and CT findings were captured, but the radiological findings had to be interpreted as their primary interpretation could not be performed retrospectively.

Statistical analysis. Response data were analyzed using descriptive statistical methods. The results are reported as the mean and standard deviation for continuous data and counts and percentages for categorical data. The PFS curves were estimated using the Kaplan-Meier method, and sub-group analyses of treatment responses were carried out by baseline characteristics using 95\% CI and logistic regression. All comparative analyses are performed at significance level 0.05. All analyses were carried out using the SAS 9.3 software version (SAS Institute, Cary, NC, USA).

\section{Results}

Demographic and baseline characteristics. During May 2008 to July 2013, the study identified 60 patients with NSCLC Stage IV, of these, 27 patients were excluded from the study for reasons such as gefitinib not being prescribed (n=3), EGFR mutation negative $(n=10)$, or missing data $(n=14)$. A total of 33 patients with median age 58.0 (32.0-77.0) years were included in the study, of these, $32(97.0 \%)$ patients had adenocarcinoma and 1 (3\%) had adenosquamous carcinoma. All the patients received gefitinib $250 \mathrm{mg}$ /day orally until disease progression occurred. The median treatment duration was 10.9 months $( \pm 11.6)$. Treatment remains ongoing in 5 patients, and 2 patients were lost to follow-up. The details of population characteristics are presented in Table I.

Response and survival. A total of 18 (54.5\%) and 2 (6.1\%) patients achieved PR and CR to gefitinib therapy, respectively. An ORR of $60.6 \%$ (95\% CI, 42.1-77.1\%) was achieved. A total of $5(15.2 \%)$ patients each achieved SD and PD. A total of 2 patients with CR were non-smoker female adenocarcinoma patients with EGFR mutation in exon 19. Meanwhile, patients with exon 20 or 21 mutations $(n=6,66.7 \%)$ tended to have improved ORR compared with exon $19(n=22,59.1 \%)$, although may be due to unequal sample size. In 4 patients, the response could not be assessed due to missing data (Table II).

It is noteworthy that there were 5 patients in the study that develops brain metastasis, of which, 4 achieved PR and 1 achieved SD. On an interim assessment, it was observed that the brain metastatic focus disappeared in 1 patient with PR. At the time of analysis, the median PFS was 8.9 months (Fig. 1). Univariate analysis revealed that an Eastern Cooperative 


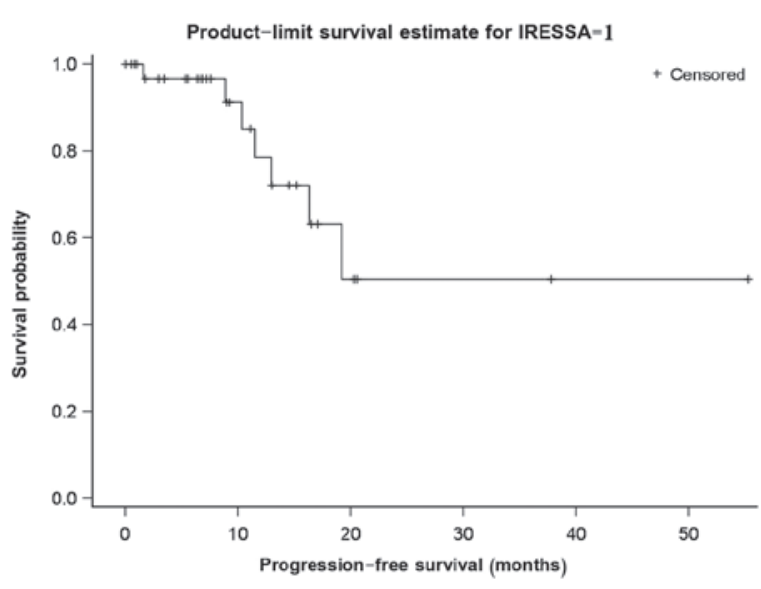

Figure 1. Kaplan-meier curve for progression free survival.

Oncology Group (ECOG) performance status of 0 or 1 was significantly associated with a longer PFS.

Drug safety and toxicity. Table III lists the adverse events based on severity. The majority of the treatment-related toxicity was mild in nature (National Cancer Institute-Common Toxicity Criteria grade 1 or 2). Missing toxicity information of patients was reported conservatively with grade 3 (severe) toxicity level. The most frequently reported AEs of grade 1 or 2 included dry skin (39.4\%), skin rash $(27.2 \%)$, and dermatitis acneiform $(15.2 \%)$. One patient developed progressive dyspnea due to pneumonitis, which was severe in nature. Symptomatic treatment was given following leading to patient improvement, and was eventually switched over to erlotinib. Other toxicities were generally tolerable, and no unexpected toxicities were observed.

\section{Discussion}

The results of this retrospective study conducted in Malaysian patients with EGFR-positive NSCLC, demonstrated that gefitinib therapy is effective in this population, as assessed by ORR of $60.6 \%$ and median PFS of 8.9 months. Gefitinib therapy was well tolerated, with patients reporting mild-to-moderate skin toxicity, and AEs consistent with the characterized tolerability/safety profile for gefitinib $(8-10,13)$. These findings revealed that gefitinib therapy was associated with improved outcomes in EGFR-positive NSCLC in Malaysian patients. The findings of this study were consistent with results from the IPASS study (6), and previous IRESSA studies conducted in the relapsed setting and underlying disease; the most commonly reported AE with IRESSA in these studies being mild-to-moderate rash and diarrhea $(8,10,12)$.

The percentage of patients in IRESSA Dose Evaluation in Advanced Lung Cancer study 1 and 2 (IDEAL 1 and IDEAL 2), and IRESSA Survival Evaluation in Lung Cancer (ISEL) study with adenocarcinoma were only 61.5, 68.6, and $47.9 \%$, respectively (13-15), whereas in the present study, $97 \%$ of the patients had adenocarcinoma, with $>50 \%$ of the patients being treatment naive. Adenocarcinoma histology, East-Asian ethnicity, female gender, and no smoking history are all clinical predictive factors of response in patients treated with gefitinib $(5,6)$; of note, $\geq 50 \%$ of the patients recruited in
Table I. Patient baseline characteristics.

\begin{tabular}{|c|c|}
\hline Characteristics & Overall $(n=33)$ \\
\hline \multicolumn{2}{|l|}{ Gender, n $(\%)$} \\
\hline Female & $29(87.9 \%)$ \\
\hline Male & $4(12.1 \%)$ \\
\hline \multicolumn{2}{|l|}{ Age (years) } \\
\hline Mean (SD) & $57.1(11.7)$ \\
\hline Median (Range) & $60.0(32.0-77.0)$ \\
\hline \multicolumn{2}{|l|}{ Age category (years), n (\%) } \\
\hline 60 years and below & $18(54.5 \%)$ \\
\hline Above 60 years & $15(45.5 \%)$ \\
\hline \multicolumn{2}{|l|}{ ECOG performance status, n (\%) } \\
\hline 0 & $8(24.2 \%)$ \\
\hline 1 & $20(60.6 \%)$ \\
\hline 2 & $4(12.1 \%)$ \\
\hline 3 & $1(3.0 \%)$ \\
\hline \multicolumn{2}{|l|}{ Disease condition, $\mathrm{n}(\%)$} \\
\hline Adenocarcinoma & $32(97.0 \%)$ \\
\hline Adenosquamous carcinoma & $1(3.0 \%)$ \\
\hline \multicolumn{2}{|l|}{ Disease stage, n (\%) } \\
\hline IV & $33(100.0 \%)$ \\
\hline \multicolumn{2}{|l|}{ Smoking history, n (\%) } \\
\hline Current/former & $3(9.1 \%)$ \\
\hline Non-smoker & $30(90.9 \%)$ \\
\hline \multicolumn{2}{|l|}{ Significant PMH/FH, n (\%) } \\
\hline No & $14(42.4 \%)$ \\
\hline Yes & $19(57.6 \%)$ \\
\hline \multicolumn{2}{|l|}{ EGFR mutation, $\mathrm{n}(\%)$} \\
\hline $\begin{array}{l}\text { Detected [15bp del (nt2235-2249) } \\
\text { CD 746-750] in EXON } 19\end{array}$ & $2(6.1 \%)$ \\
\hline $\begin{array}{l}\text { Detected [15bp del (nt2236-2250) } \\
\text { CD 746-750] in EXON } 19\end{array}$ & $1(3.0 \%)$ \\
\hline Detected [Deletion] in EXON 19 & $18(54.5 \%)$ \\
\hline $\begin{array}{l}\text { Detected [Deletion] in EXON 19, } \\
\text { [Insertion] in EXON } 20 \text { and } \\
\text { [L858R] in EXON } 21\end{array}$ & $1(3.0 \%)$ \\
\hline Detected [L858R] in EXON 21 & $3(9.1 \%)$ \\
\hline $\begin{array}{l}\text { Detected [T to G (NT 2573) } \\
\text { L858R] in EXON } 21\end{array}$ & $2(6.1 \%)$ \\
\hline $\begin{array}{l}\text { Detected [T790M] in EXON } 20 \text { and } \\
\text { [L858R] in EXON } 21\end{array}$ & $1(3.0 \%)$ \\
\hline Mutation detected & $5(15.2 \%)$ \\
\hline
\end{tabular}

ECOG, eastern cooperative oncology group; EGFR, epidermal growth factor receptor; $\mathrm{FH}$, family history; $\mathrm{PMH}$, past medical history; SD, standard deviation.

the present study had these baseline characteristics. A correlation between specific EGFR mutations and tumor response to gefitinib was demonstrated by Lynch et al (16). Furthermore, an ORR of $71.2 \%$ was observed with first-line gefitinib treatment in patients with EGFR mutation-positive adenocarcinoma in 


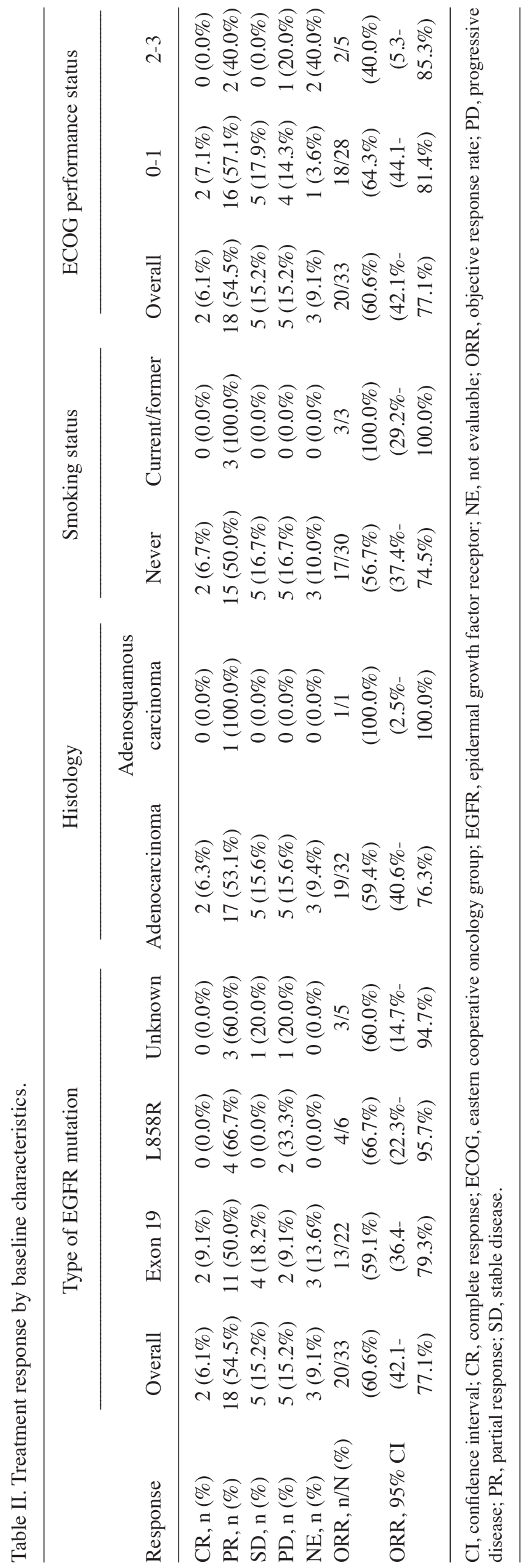


Table III. Summary of patients with adverse events by severity.

\begin{tabular}{|c|c|c|c|c|}
\hline AEs & Mild & Moderate & Severe & Overall \\
\hline Dermatitis acneiform $^{a}$ & $5(15.2 \%)$ & $1(3.0 \%)$ & $0(0.0 \%)$ & $4(12.1 \%)$ \\
\hline Diarrhea & $1(3.0 \%)$ & $0(0.0 \%)$ & $0(0.0 \%)$ & $1(3.0 \%)$ \\
\hline Dry mouth & $1(3.0 \%)$ & $0(0.0 \%)$ & $0(0.0 \%)$ & $1(3.0 \%)$ \\
\hline Dry skin ${ }^{b}$ & $13(39.4 \%)$ & $0(0.0 \%)$ & $0(0.0 \%)$ & $12(36.4 \%)$ \\
\hline In-growing nail & $1(3.0 \%)$ & $0(0.0 \%)$ & $0(0.0 \%)$ & $1(3.0 \%)$ \\
\hline Koilonychias & $1(3.0 \%)$ & $0(0.0 \%)$ & $0(0.0 \%)$ & $1(3.0 \%)$ \\
\hline Mouth ulceration & $1(3.0 \%)$ & $0(0.0 \%)$ & $0(0.0 \%)$ & $1(3.0 \%)$ \\
\hline Onychalgia & $1(3.0 \%)$ & $0(0.0 \%)$ & $0(0.0 \%)$ & $1(3.0 \%)$ \\
\hline Pneumonitis, dyspnea & $0(0.0 \%)$ & $0(0.0 \%)$ & $1(3.0 \%)$ & $1(3.0 \%)$ \\
\hline $\operatorname{Rash}^{\mathrm{c}}$ & $6(21.2 \%)$ & $0(0.0 \%)$ & $1(3.0 \%)$ & $4(12.1 \%)$ \\
\hline Erythematous rash & $1(3.0 \%)$ & $0(0.0 \%)$ & $0(0.0 \%)$ & $1(3.0 \%)$ \\
\hline Maculopapular rash & $1(3.0 \%)$ & $0(0.0 \%)$ & $0(0.0 \%)$ & $1(3.0 \%)$ \\
\hline Xerosis & $2(6.0 \%)$ & $0(0.0 \%)$ & $0(0.0 \%)$ & $1(3.0 \%)$ \\
\hline
\end{tabular}

AE, adverse event. Patients with multiple AEs were not included for evaluation in the overall population. ${ }^{2} 2$ patients had Dermatitis acneiform along with rash. ${ }^{\mathrm{b}} 1$ patient had dry skin along with rash. ${ }^{\mathrm{c}} 1$ patient with dry skin, 2 patients with dermatitis acneiform and 1 patient with xerosis had rash.

the IRESSA Pan-Asia Study (IPASS) (6). In the present study, a similar but slightly lower ORR of $60.6 \%$ was observed, even though all the patients were EGFR mutation-positive, maybe because of the small sample size, patients lost to follow-up or patients not included in the analysis because of incomplete data. The median survival was shown to be improved to over 2 years with gefitinib in mutation-positive NSCLC patients in the IRESSA Combined Analysis of the Mutation Positives study (17). In a small sub-group of 44 EGFR mutation-positive patients, an improved PFS and higher RR were seen with gefitinib than docetaxel (18). Liam et al reported that 26 patients (36.6\%) achieved PR whereas no patient had CR. Moreover, 26 (36.6\%) and 19 (26.8\%) patients had SD and PD, respectively, with gefitinib treatment (11). A small retrospective analysis also revealed a $48 \%$ PR and $13 \%$ SD in a Malaysian population treated with gefitinib (12). It can be inferred from these studies that gefitinib offers good efficacy in Asian EGFR mutation-positive patients; hence, EGFR mutation status must be determined before starting treatment with gefitinib especially in a first-line setting. The proportion of non-smokers who responded to gefitinib was more than double compared with those who had smoked, due to the small sample size. A higher RR has been observed in non-smokers in previous studies $(11,12)$. A good ECOG performance status of $0-1$ independently predicted the response to gefitinib monotherapy in our patients. Poor performance status has been identified as a predictor of poor response to gefitinib $(13,19)$.

In a trial with 41 Japanese patients with brain metastases from $E G F R$-mutant lung adenocarcinoma, an ORR of $87.8 \%$ and a median PFS of 14.5 months were observed with gefitinib monotherapy without radiation (20). In our study, 4 patients with brain metastases achieved PR with gefitinib, and 1 patient achieved SD. The AEs of gefitinib therapy in the patients in the present study were generally mild and consisted mainly of skin reactions as has been reported by others (13). The incidence of diarrhea was relatively uncommon as observed with other studies (5). Only 1 patient in the present study reported severe pneumonitis leading to dyspnea, which was symptomatically treated and gefitinib treatment was stopped.

The present study has several limitations. Due to its retrospective nature, access to medical data was unimpeded. However, the recordings of observation were not standardized, and often there were missing data. The timing of scans to assess response was also not uniform amongst the clinicians. The sample size was small and the study was conducted in a single tertiary center in Malaysia, making it difficult to extrapolate these results.

In conclusion, this retrospective analysis demonstrated that Malaysian patients with locally advanced and metastatic EGFR mutation-positive lung adenocarcinoma responded favorably to gefitinib therapy in terms of ORR, median PFS, and tolerability, the results of which were consistent with IPASS study conducted in Asian population. Considering the efficacy and safety profile of gefitinib, it is a favorable option for the first-line treatment of Malaysian patients with EGFR mutation-positive NSCLC. However, future long-term studies in larger population of Malaysian patients are required to support whether the prolonged PFS conferred by gefitinib will translate into prolonged overall survival.

\section{Conflict of interest}

The present study was funded by AstraZeneca. IRESSA (gefitinib) is a trademark of the AstraZeneca group of companies. Dr Matin Mellor Abdullah received honorarium from Astra Zeneca for the previous engagement.

\section{References}

1. Liam CK, Pang YK, Leow CH, et al: Changes in the distribution of lung cancer cell types and patient demography in a developing multiracial Asian country: Experience of a university teaching hospital. Lung Cancer 53: 23-30, 2006. 
2. NationalCancerRegistry (2007). Malaysia Cancer Statistics - Data and Figure, National Cancer Registry, Malaysia. Available at URL: http://www.care.upm.edu.my/dokumen/13603_NCR2007. pdf. Accessed September 8, 2014.

3. National Comprehensive Cancer Network: 2011 NCCN Clinical Practice Guidelines in Oncology: Non-small Cell Lung Cancer, Version 1. National Comprehensive Cancer Network (2011) NCCN Clinical Practice Guidelines in Oncology 2011; Available at: URL: http://www.ncen.org/patients/guidelines/nscl/index. html. Accessed September 8, 2014.

4. Liam CK, Leow HR, How SH, Pang YK, Chua KT, Lim BK, Lai NL, Kuan YC, Pailoor J and Rajadurai P: Epidermal growth factor receptor mutations in non-small cell lung cancers in a multiethnic malaysian patient population. Asian Pac J Cancer Prev 15: 321-326, 2014

5. Ho C, Murray N, Laskin J, Melosky B, Anderson H and Bebb G: Asian ethnicity and adenocarcinoma histology continues to predict response to gefitinib in patients treated for advanced non-small cell carcinoma of the lung in North America. Lung Cancer 49: 225-231, 2005.

6. Mok TS, Wu YL, Thongprasert S, Yang CH, Chu DT, Saijo N, Sunpaweravong P, Han B, Margono B, Ichinose Y, et al: Gefitinib or carboplatin-paclitaxel in pulmonary adenocarcinoma. N Engl J Med 361: 947-957, 2009.

7. Mitsudomi T, Morita S, Yatabe Y, Negoro S, Okamoto I, Tsurutani J, Seto T, Satouchi M, Tada H, Hirashima T, et al: Gefitinib versus cisplatin plus docetaxel in patients with non-small-cell lung cancer harbouring mutations of the epidermal growth factor receptor (WJTOG3405): An open label, randomised phase 3 trial. Lancet Oncol 11: 121-128, 2010.

8. Maemondo M, Inoue A, Kobayashi K, Sugawara S, Oizumi S, Isobe H, Gemma A, Harada M, Yoshizawa H, Kinoshita I, et al: Gefitinib or chemotherapy for non-small-cell lung cancer with mutated EGFR. N Engl J Med 362: 2380-2388, 2010.

9. Yang CH, Yu CJ, Shih JY, Chang YC, Hu FC, Tsai MC, Chen KY, Lin ZZ, Huang CJ, Shun CT, et al: Specific EGFR mutations predict treatment outcome of stage IIIB/IV patients with chemotherapy-naive non-small-cell lung cancer receiving first-line gefitinib monotherapy. J Clin Oncol 26: 2745-2753, 2008.

10. Chang A, Parikh P, Thongprasert S, Tan EH, Perng RP, Ganzon D, Yang $\mathrm{CH}$, Tsao CJ, Watkins $\mathrm{C}$, Botwood $\mathrm{N}$ and Thatcher $\mathrm{N}$ : Gefitinib (IRESSA) in patients of Asian origin with refractory advanced non-small cell lung cancer: Subset analysis from the ISEL study. J Thorac Oncol 1: 847-855, 2006.

11. Liam CK, Ruthranesan M, Lee CH, Pang YK, Chua KT and Lim BK: Outcomes of Malaysian patients with advanced lung adenocarcinoma and unknown epidermal growth factor receptor mutation status treated with gefitinib. Asia Pac J Clin Oncol 8: 267-274, 2012
12. Liam CK, Pang YK and Leow CH: Epidermal growth factor receptor targeted therapy with gefitinib in locally advanced and metastatic primary lung adenocarcinoma. Respirology 11: 287-291, 2006.

13. Fukuoka M, Yano S, Giaccone G, Tamura T, Nakagawa K, Douillard JY, Nishiwaki Y, Vansteenkiste J, Kudoh S, Rischin D, et al: Multi-institutional randomized phase II trial of gefitinib for previously treated patients with advanced non-small-cell lung cancer (The IDEAL 1 Trial) [corrected]. J Clin Oncol 21: 2237-2246, 2003

14. Kris MG, Natale RB, Herbst RS, Lynch TJ Jr, Prager D, Belani CP, Schiller JH, Kelly K, Spiridonidis H, Sandler A, et al: Efficacy of gefitinib, an inhibitor of the epidermal growth factor receptor tyrosine kinase, in symptomatic patients with non-small cell lung cancer: A randomized trial. JAMA 290: 2149-2158, 2003

15. Thatcher N, Chang A, Parikh P, Rodrigues Pereira J, Ciuleanu T, von Pawel J, Thongprasert S, Tan EH, Pemberton K, Archer V and Carroll K: Gefitinib plus best supportive care in previously treated patients with refractory advanced non-small-cell lung cancer: Results from a randomised, placebo-controlled, multicentre study (Iressa Survival Evaluation in Lung Cancer). Lancet 366: 1527-1537, 2005.

16. Lynch TJ, Bell DW, Sordella R, Gurubhagavatula S, Okimoto RA, Brannigan BW, Harris PL, Haserlat SM, Supko JG, Haluska FG, et al: Activating mutations in the epidermal growth factor receptor underlying responsiveness of non-small-cell lung cancer to gefitinib. N Engl J Med 350: 2129-2139, 2004.

17. Morita S, Okamoto I, Kobayashi K, Yamazaki K, Asahina H, Inoue A, Hagiwara K, Sunaga N, Yanagitani N, Hida T, et al: Combined survival analysis of prospective clinical trials of gefitinib for non-small cell lung cancer with EGFR mutations. Clin Cancer Res 15: 4493-4498, 2009.

18. Kim ES, Hirsh V, Mok T, Socinski MA, Gervais R, Wu YL, Li LY, Watkins CL, Sellers MV, Lowe ES, et al: Gefitinib versus docetaxel in previously treated non-small-cell lung cancer (INTEREST): A randomised phase III trial. Lancet 372: 1809-1818, 2008.

19. Miller VA, Kris MG, Shah N, Patel J, Azzoli C, Gomez J, Krug LM, Pao W, Rizvi N, Pizzo B, et al: Bronchioloalveolar pathologic subtype and smoking history predict sensitivity to gefitinib in advanced non-small-cell lung cancer. J Clin Oncol 22: 1103-1109, 2004.

20. Iuchi T, Shingyoji M, Sakaida T, Hatano K, Nagano O, Itakura M, Kageyama H, Yokoi S, Hasegawa Y, Kawasaki K and Iizasa T: Phase II trial of gefitinib alone without radiation therapy for Japanese patients with brain metastases from EGFR-mutant lung adenocarcinoma. Lung Cancer 82: 282-287, 2013. 